OPEN ACCESS

Edited by:

Colette Joy Browning,

Federation University, Australia

Reviewed by:

Margo Bergman

University of Washington Tacoma

United States

Dianne Goeman

University of Newcastle, Australia

${ }^{*}$ Correspondence:

Hirono Ishikawa

hirono-tky@umin.ac.jp

Specialty section:

This article was submitted to

Public Health Education and

Promotion,

a section of the journa

Frontiers in Public Health

Received: 14 March 2019

Accepted: 06 June 2019

Published: 19 June 2019

Citation:

Ishikawa H and Kiuchi T (2019) Association of Health Literacy Levels

Between Family Members.

Front. Public Health 7:169.

doi: 10.3389/fpubh.2019.00169

\section{Association of Health Literacy Levels Between Family Members}

\author{
Hirono Ishikawa ${ }^{1 *}$ and Takahiro Kiuchi ${ }^{2}$ \\ ${ }^{1}$ Teikyo University Graduate School of Public Health, Tokyo, Japan, ${ }^{2}$ Department of Health Communication, \\ Graduate School of Medicine, The University of Tokyo, Tokyo, Japan
}

Background: Health literacy $(H L)$ is not solely an individual skill but a distributed resource available within individual's social networks. This study explored the associations between individual and family member $\mathrm{HL}$ using two separate self-report measures of HL: the European Health Literacy Survey Questionnaire (HLS-EU-Q47) and the Communicative and Critical Health Literacy scale (CCHL).

Methods: A self-administered questionnaire survey was conducted with 501 pairs of Japanese residents aged 30 to 79 and their family members whom they most often consulted for help with health issues. HL was measured using HLS-EU-Q47 and CCHL.

Results: The $\mathrm{HL}$ scores of individuals and their family members were positively correlated for both measures. The correlation was stronger for the HLS-EU-Q47, presumably because it measures the perceived manageability of health-related tasks that implicitly depend on the availability of support for an individual. In contrast, the CCHL measures a single individual's perceived abilities. Both individual and family member $\mathrm{CCHL}$ scores were independently related to individual HLS-EU-Q47 scores, particularly when an individual had a family member with a higher CCHL score than his/her own.

Conclusions: Limited individual ability to achieve health-related tasks might be compensated for by the higher ability of other family members. In addressing problems with limited health literacy, future studies should focus not only on the individual but also on people who can provide an individual with support.

\footnotetext{
Keywords: health literacy, family, social support, HLS-EU-Q47 (The European Health Literacy Survey Questionnaire), $\mathrm{CCHL}$ (Communicative and Critical Health Literacy scale)
}

\section{INTRODUCTION}

Over the past few decades, health literacy (HL) has gained importance, as it is a factor related to health behaviors and outcomes. HL represents "the cognitive and social skills which determine the motivation and ability of individuals to gain access to, understand and use information in ways which promote and maintain good health" (1). It is the achievement of a level of knowledge, confidence, and personal skills that enable an individual to take actions to improve personal and community health by changing personal lifestyles and living conditions.

In light of the growing evidence of the relationship between inadequate $\mathrm{HL}$ and poor health outcomes, research has been undertaken into possible interventions to increase HL (2-7). These studies provide evidence that individuals with lower HL can be identified and thus helped to develop skills and a better understanding of their health conditions (8). 
However, certain patients with chronic diseases successfully manage their conditions despite poor scores on HL and knowledge (9). Valuable resources and support from one's social networks seem to buffer and alleviate the adverse consequences of low HL (10). Thus, HL is not solely an individual skill but a distributed resource available within an individual's social entourage (11). Building on this conclusion, a prior study revealed that HL was distributed among family and social networks and that individuals often depended on the HL skills of others to understand and use health information (12). Qualitative interviews with individuals with long-term health conditions helped develop a HL pathway model that highlighted how the support of family, friends, and health professionals facilitated the development of HL. These entities support HL by helping with health-related tasks and facilitating an individual's active involvement in the management of his or her health. This may be particularly true in collectivist Asian cultures (13).

A measure of comprehensive HL, the European Health Literacy Survey Questionnaire (HLS-EU-Q47), was developed to measure HL in populations (14) based on a conceptual framework reflecting four information-processing dimensions (i.e, accessing, understanding, appraising, and applying) within three health domains (i.e, health care, disease prevention, and health promotion) (15). Each item in the questionnaire assesses the perceived difficulty of a specific health-related task for the respondent. It has been used and validated internationally in Japan and other Asian countries (16, 17). The HLS-EU-Q47 is unique in that it measures the subjective manageability of health-related tasks, both focusing on the individual and considering the underlying circumstances in which health-related tasks are performed $(18,19)$. In this sense, it implicitly reflects the support available to an individual.

Another HL scale that measures beyond functional HL in the general population was developed and validated in Japan (20). The Communicative and Critical Health Literacy scale (CCHL) is based on a model of the three dimensions of HL (i.e, functional/basic, communicative/interactive, and critical health literacy) (21). It assesses the perceived ability of the respondent to find and utilize health and medical information as required. Although the conceptual components are similar to the HLS-EUQ47, it assesses respondents' ability to complete health-related tasks on their own.

A previous study reported moderate correlations $(r=0.55-$ 0.62) between HLS-EU-Q47 and CCHL scores (17). However, no further studies have explored this relationship. Few studies have examined the relationship between an individual's HL and that of the people close to that individual. We explored the association between the HL of individuals and their family members using self-report scales of HL. More specifically, we explored the following:

1) Whether individual HL and that of family members are positively related. We expected that the correlation would be higher for the HLS-EU-Q47, which measures the subjective manageability of health-related tasks, than for the CCHL, which measures a respondent's own perceived ability.
2) Whether both individual's and family member's CCHL scores are related to individual's HLS-EU-Q47.

3) Whether the relationship between individual's and family member's CCHL score and individual's HLS-EU-Q47 score would differ by the individual's CCHL score in relation to the family member's CCHL score. For those who have a family member with higher CCHL than themselves, the family member's CCHL would be more important than for those with higher CCHL than the family member

\section{METHODS}

\section{Participants and Study Procedures}

Participants were recruited from a pool of Japanese residents provided by a survey research company database. From the database, we aimed to recruit 500 Japanese residents ages 30 to 79 who lived with their families. Respondents who met the inclusion criteria were randomly invited to participate via fax/mail. They were asked to participate together with the family member they most often consulted for help with health issues. Those who agreed to participate in the survey were asked to provide a completed consent form. We attempted to match participants according to gender and age according to Japanese population data from 2016. Responses from potential participants were collected up to the targets set for each gender and age group.

A set of self-administered questionnaires was mailed to both individuals and their respective family members; questionnaires were to be completed independently. In total, data were collected from 501 pairs. This study was approved by the ethical review committee at the University of Tokyo Graduate School of Medicine (approval no. 11476).

\section{Measures}

\section{Health Literacy}

The HLS-EU-Q47 was translated and validated in a previous study in a Japanese population (17). The scale contains 47 items that measure HL. Each item assesses the perceived difficulty of completing a specific health-related task by asking "On a scale from very difficult to very easy, how easy would you say it is to [e.g, find information on symptoms of illnesses that concern you]?" Each item was rated on a 4-point Likert-type scale (1, very difficult; 2 , fairly difficult; 3 , fairly easy; 4 , very easy). A higher difficulty score was assumed with lower HL. Using scores from all 47 questionnaire items, we constructed a comprehensive general index of HL. Following the original study, a mean-based item raw score was computed for respondents who gave valid answers to at least $80 \%$ of all HL questions (19). The index score was standardized to unified metrics from 0 to 50 using the following formula: $(\mathrm{MEAN}-1) \times(50 / 3)$. Responses of “don't know" were treated as missing and not included in calculations of participants' index scores (17). Cronbach's alpha for the scale was 0.96 .

HL was also measured using the Communicative and Critical Health Literacy Scale (20). This scale is based on an established model of HL (21) and consists of five items addressing whether the respondent himself or herself is able to (1) collect health information from various sources, (2) extract relevant 
information, (3) understand and communicate the information obtained, (4) consider the credibility of the information, and (5) make decisions based on the information in the context of health issues. Each item is scored on a 5-point scale ranging from strongly disagree (1) to strongly agree (5). Scores for the items in each scale were summed and divided by the number of items in that scale to yield a scaled score (theoretical range $=1-5$ ). Cronbach's alpha for the scale was 0.84 .

\section{Sociodemographics}

The following demographic data were also obtained as part of the survey: age (in years), gender (male or female), educational attainment (junior high school, high school/vocational school, 2year college, university or more), self-assessed economic status (on a 10-point scale ranging from $1=$ lowest to $10=$ highest in society), and having a currently treated disease (yes/no).

\section{Statistical Analyses}

Bivariate associations between individual and family HL scores were examined using Pearson correlation analysis. Individuals were divided into one of two groups based on their CCHL score in relation to their family member's CCHL score: (1) those with a higher CCHL score than their family member and (2) those with the same or a lower CCHL score than their family member. Differences in sociodemographic characteristics between these two groups were examined using chi-square, Kruskal-Wallis, or independent $t$-tests. Regression analyses of individual and family member CCHL scores on HLS-EU-Q47 scores were performed controlling for sociodemographic variables, and subsequently thorough analyses were performed on each group. All data were analyzed using Stata 14.2 (StataCorp, College Station, TX, USA).

\section{RESULTS}

The study included a total of 501 individuals and 501 family members identified by the individuals as the people they most often consulted about their health. Table 1 displays the sociodemographic characteristics and HL scores of the individuals and their family members. The mean age of the individuals was 54.1 years $(S D=13.7)$, and $49.3 \%$ were male. The average age of the family members was 57.6 years old $(S D=13.4$, range $=14-95)$, and there were more women than men ( $60.7 \%$ female, $39.3 \%$ male). As expected, the majority of the family members were spouses of the individual study participants (72.7\%), followed by mothers (16.4\%).

The mean HL scores for the individuals and their family members were $30.14(S D=7.46)$ and $30.29(S D=8.13)$ based on the HLS-EU-Q47. CCHL scores were $3.61(S D=0.66)$ and $3.50(S D=0.77)$, respectively.

As shown in Table 2, in 228 pairs (45.5\%) the individual had a higher CCHL score than the family member, whereas in 273 pairs $(54.5 \%)$ the family member's CCHL score was the same or higher. No statistically significant differences existed between the two groups in demographic characteristics.
TABLE 1 | Descriptive statistics for the participants.

\begin{tabular}{|c|c|c|c|c|c|}
\hline & & \multicolumn{2}{|c|}{ Individuals } & \multicolumn{2}{|c|}{ Family } \\
\hline & & $N$ & $\%$ & $N$ & $\%$ \\
\hline \multirow[t]{7}{*}{ Age } & $14-29$ & - & - & 13 & 2.6 \\
\hline & 30-39 & 98 & 19.6 & 15 & 3.0 \\
\hline & $40-49$ & 114 & 22.8 & 118 & 23.6 \\
\hline & $50-59$ & 94 & 18.8 & 119 & 23.8 \\
\hline & $60-69$ & 111 & 22.2 & 161 & 32.1 \\
\hline & $70-79$ & 84 & 16.8 & 75 & 15.0 \\
\hline & Mean (SD) & 54.1 & $(13.7)$ & 57.6 & (13.4) \\
\hline \multirow[t]{2}{*}{ Gender } & Male & 247 & 49.3 & 197 & 39.3 \\
\hline & Female & 254 & 50.7 & 304 & 60.7 \\
\hline \multirow{4}{*}{$\begin{array}{l}\text { Educational } \\
\text { attainment }\end{array}$} & Junior high school & 22 & 4.4 & 30 & 6.0 \\
\hline & $\begin{array}{l}\text { High school/ } \\
\text { Vocational school }\end{array}$ & 210 & 41.9 & 243 & 48.5 \\
\hline & 2-year college & 65 & 13.0 & 75 & 15.0 \\
\hline & University and above & 204 & 40.7 & 153 & 30.5 \\
\hline \multirow{6}{*}{$\begin{array}{l}\text { Relationship with } \\
\text { the individual }\end{array}$} & Spouse & & & 364 & 72.7 \\
\hline & Mother & & & 82 & 16.4 \\
\hline & Father & & & 13 & 2.6 \\
\hline & Daughter & & & 26 & 5.2 \\
\hline & Son & & & 6 & 1.2 \\
\hline & Other & & & 10 & 1.9 \\
\hline \multirow{5}{*}{$\begin{array}{l}\text { Self-assessed } \\
\text { economic status }\end{array}$} & Low:1-3 & 75 & 15.0 & & \\
\hline & Middle low: 4-5 & 205 & 40.9 & & \\
\hline & Middle high: 6-7 & 194 & 38.7 & & \\
\hline & High: 8-10 & 23 & 4.6 & & \\
\hline & missing & 4 & 0.8 & & \\
\hline \multirow{3}{*}{$\begin{array}{l}\text { Having a currently } \\
\text { treated disease }\end{array}$} & Yes & 268 & 53.5 & & \\
\hline & No & 222 & 44.3 & & \\
\hline & missing & 11 & 2.2 & & \\
\hline \multicolumn{6}{|l|}{ Health literacy } \\
\hline HLS-EU-Q47 & Mean (SD) & 30.14 & $(7.46)$ & 30.29 & (8.13) \\
\hline $\mathrm{CCHL}$ & Mean (SD) & 3.61 & $(0.66)$ & 3.50 & $(0.77)$ \\
\hline
\end{tabular}

\section{Correlations Between Individual and Family Member Health Literacy}

As shown in Table 3, HL scores of the individuals and their family members were positively correlated for both the HLS-EU-Q47 and the CCHL. The correlation was much stronger for HLS-EUQ47 than for CCHL scores $(r=0.490, p<0.001$, and $r=0.252$, $p<0.001$, respectively).

Correlations between the two HL scores (i.e, HLS-EU-Q47 and CCHL scores) were similar for individuals $(r=0.434$, $p<0.001)$ and family members $(r=0.590, p<0.001)$.

\section{Relationships of Individual and Family Member CCHL Scores With Individual HLS-EU-Q47 Scores}

Table 4 shows regression analyses of individual and family member CCHL scores on individual HLS-EU-Q47 scores controlling for sociodemographic variables. In the total sample, 
both individual and family member CCHL scores were positively related to individual HLS-EU-Q47 scores. Age and economic status were positively associated with the HLS-EU-47 score.

Among individuals with a higher CCHL score than their family member (individual CCHL > family member CCHL), only individual CCHL score, not family CCHL score, was significantly correlated with the HLS-EU-Q47 score. By contrast, both individual and family member CCHL scores were related to HLS-EU-Q47 scores among individuals with a lower CCHL score than their family member (individual CCHL $\leq$ family CCHL).

\section{DISCUSSION}

This study examined the association between individual and family member HL using two different measures of HL: the HLS-EU-Q47 and the CCHL. Our hypotheses were generally supported by our survey data.

The HL scores of individuals and their family members were positively correlated for both measures. As expected, the correlation was much greater for the HLS-EU-Q47 than for the CCHL. Although the HLS-EU-Q47 is more comprehensive and covers more components of HL, the theoretical framework of the two measures is similar. Both questionnaires were developed to measure the HL of non-patient populations, not only in the medical/clinical context but also in the public health context. Both measure HL beyond the functional level, focusing on the ability to access, understand, and utilize health information $(14,20)$. A major difference between the two measures is that the HLS-EU-Q47 measures the perceived difficulty of achieving health-related tasks, whereas the CCHL measures the perceived ability of the respondent to complete tasks. As reported in a previous study (17), HLS-EU-Q47 and CCHL scores were positively correlated with each other, but the correlation was not that strong. Thus, compared to the CCHL score, the HLS-EUQ47 score more directly reflects the support available from other people in an individual's network in addition to the individual's ability and resulted in higher correlations between individual and family member scores.

Both individual and family member CCHL scores (one's perceived ability to do health-related tasks) were independently related to the individual HLS-EU-Q47 score (perceived manageability of health-related tasks). The HL of an individual depends on the population to which he or she belongs (22). Higher population HL attenuates the demand for individual HL by communicating health information in appropriate ways but also moderates the relationship between an individual's HL, health behaviors, and outcomes by providing support for the individual to seek and understand health information,

TABLE 3 | Correlations between individual and family member health literacy.

\begin{tabular}{lccc}
\hline & $\boldsymbol{N}^{\mathbf{a}}$ & $\boldsymbol{r}^{\mathbf{b}}$ & $\boldsymbol{p}$-value \\
\hline Individual and Family CCHL & 501 & 0.252 & $<0.001$ \\
Individual and Family HLS-EU & 412 & 0.490 & $<0.001$ \\
Individual CCHL and HLS-EU & 450 & 0.434 & $<0.001$ \\
Family CCHL and HLS-EU & 444 & 0.590 & $<0.001$
\end{tabular}

a Listwise deletion for missing data.

${ }^{b}$ Pearson correlation coefficients.

TABLE 2 | Descriptive statistics by the groups (Individual CCHL>Family CCHL vs. Individual CCHL $\leq$ Family CCHL).

\begin{tabular}{|c|c|c|c|c|c|c|}
\hline & & \multicolumn{2}{|c|}{$\begin{array}{c}\text { Individual CCHL > Family } \\
\text { CCHL }(N=228)\end{array}$} & \multicolumn{2}{|c|}{$\begin{array}{c}\text { Individual } \mathrm{CCHL} \leq \text { Family } \\
\mathrm{CCHL}(N=273)\end{array}$} & \multirow[b]{2}{*}{$p$-value } \\
\hline & & $\mathbf{N}$ & $\%$ & $\mathbf{N}$ & $\%$ & \\
\hline Individual CCHL & Mean (SD) & 3.85 & 0.58 & 3.40 & 0.65 & \\
\hline Family CCHL & Mean (SD) & 3.02 & 0.76 & 3.89 & 0.51 & \\
\hline Age & Mean (SD) & 53.7 & 13.2 & 54.4 & 14.2 & $0.605^{a}$ \\
\hline \multirow[t]{2}{*}{ Gender } & Male & 124 & 45.4 & 123 & 54.0 & $0.057^{b}$ \\
\hline & Female & 149 & 54.6 & 105 & 46.1 & \\
\hline \multirow[t]{4}{*}{ Educational attainment } & Junior high school & 10 & 4.4 & 12 & 4.4 & $0.950^{C}$ \\
\hline & $\begin{array}{l}\text { High school/ Vocational } \\
\text { school }\end{array}$ & 96 & 42.1 & 114 & 41.8 & \\
\hline & 2-year college & 28 & 12.3 & 37 & 13.6 & \\
\hline & University and above & 94 & 41.2 & 110 & 40.3 & \\
\hline \multirow[t]{4}{*}{ Self-assessed economic status } & Low:1-3 & 33 & 14.6 & 42 & 15.5 & $0.575^{\mathrm{C}}$ \\
\hline & Middle low: 4-5 & 92 & 40.7 & 113 & 41.7 & \\
\hline & Middle high: 6-7 & 88 & 38.9 & 106 & 39.1 & \\
\hline & High: 8-10 & 13 & 5.8 & 10 & 3.7 & \\
\hline Having a currently treated disease & Yes & 119 & 53.6 & 149 & 55.6 & $0.659^{b}$ \\
\hline HLS-EU-Q47 & Mean (SD) & 30.78 & 7.65 & 29.59 & 7.26 & $0.094^{a}$ \\
\hline
\end{tabular}

at-test.

${ }^{b}$ chi-square test.

${ }^{c}$ Kruskal-Wallis test. 
TABLE 4 | Relationships of individual and family CCHL with the HLS-EU-47 by the difference between individual and family CCHL.

\begin{tabular}{|c|c|c|c|c|c|c|c|c|c|}
\hline & \multicolumn{3}{|c|}{ All $(N=446)$} & \multicolumn{3}{|c|}{$\begin{array}{l}\text { Individual CCHL> Family } \\
\text { CCHL }(N=199)\end{array}$} & \multicolumn{3}{|c|}{$\begin{array}{c}\text { Individual } \mathrm{CCHL} \leq \text { Family } \\
\text { CCHL }(N=242)\end{array}$} \\
\hline & B & s.e. & $p$-value & B & s.e. & $p$-value & B & s.e. & $p$-value \\
\hline Age & 0.056 & 0.025 & 0.025 & 0.082 & 0.038 & 0.032 & 0.025 & 0.033 & 0.443 \\
\hline Gender & 0.780 & 0.654 & 0.234 & 2.607 & 0.992 & 0.009 & -0.973 & 0.869 & 0.264 \\
\hline Education & -0.423 & 0.342 & 0.217 & -0.249 & 0.512 & 0.628 & -0.599 & 0.451 & 0.185 \\
\hline Economic status & 0.914 & 0.422 & 0.031 & 0.061 & 0.636 & 0.924 & 1.619 & 0.555 & 0.004 \\
\hline Having a disease & 0.865 & 0.675 & 0.200 & 1.354 & 1.013 & 0.183 & 0.461 & 0.889 & 0.605 \\
\hline Individual CCHL & 4.621 & 0.494 & $<0.001$ & 4.997 & 0.991 & $<0.001$ & 3.301 & 0.810 & $<0.001$ \\
\hline Family CCHL & 1.221 & 0.425 & 0.004 & 1.022 & 0.763 & 0.182 & 3.454 & 1.052 & $<0.001$ \\
\hline (constant) & 3.418 & 2.825 & 0.227 & -0.047 & 4.299 & 0.991 & 2.512 & 3.964 & 0.527 \\
\hline Adjusted R-squared & 0.237 & & & 0.245 & & & 0.251 & & \\
\hline
\end{tabular}

make health decisions, and manage his or her health (23). Previous studies on the relationship between adult children's educational attainment and parents' health have suggested that individuals with higher education are more likely to communicate health knowledge to family members and to share skills that can improve health behavior and help to navigate and utilize complex health care system resources (24-26). It is widely known that the educational attainment is closely related to HL $(27,28)$. Family members with higher abilities may help other family members to do health-related tasks and thus independently contribute to better manageability of health-related tasks.

This relationship is more evident for those who have a family member with a higher CCHL than themselves, whereas only individuals' own CCHL counted for those who had a higher CCHL than their family member. We speculate that limited individual ability to achieve health-related tasks might be compensated for by the higher ability of other family members. In other words, lower individual ability is not necessarily associated with poor outcomes if there is someone with higher ability to support that individual. This finding coincides with studies that suggest that social capital attenuates the effects of limited HL on health information behaviors $(29,30)$. Social capital refers to features of social relationships such as levels of interpersonal trust and norms of reciprocity and mutual aid that facilitate collective action for mutual benefit (31). HL at the population level is closely related to the concept of social capital (32).

Previous interventions with HL have been focused on individuals with limited HL (2-7). Our findings suggest that to solve problems of limited HL, interventions should target both individuals and those who could provide support to them, such as family, relatives, and friends. Populations with higher $\mathrm{HL}$ are those in which health information is provided in an understandable way, patients are supported in making healthy decisions, social norms influence economic determinants of health, and there is strong community empowerment (33). Thus, the HL of the people surrounding an individual might indeed influence the relationship between that individual's $\mathrm{HL}$ and his or her health outcomes. However, our findings also suggest that $\mathrm{HL}$ is positively correlated among family members. To support an individual with limited HL who lives alone or is surrounded by family members with low HL, provisions should be put in place beyond the family unit involving the community, the health care system, and policy.

Limitations to our study exist. First, the participants in this study were recruited from the database of a survey research company; thus, we were unable to include individuals who were not interested in participating in such commercial surveys. It is possible that our sample was not representative of the general population of Japan. The proportion of university graduates in our sample was approximately $40 \%$, much higher than in national census data (approximately $20 \%$ in 2010). Although the proportion of university graduates was comparable to a recent Japanese study based on an online survey of HL, the mean score on the HLS-EU-Q47 was higher than in previous studies $(N=927$; mean $\pm S D, 25.3 \pm 8.2)$ (17). This is partially because only those living with their family member were included in the study. In terms of the CCHL score, the mean score of 3.61 was similar to that of a previous nationwide online survey of the general population in Japan $(N=712$; mean $\pm \mathrm{SD}, 3.59 \pm 0.62)(34)$. But it was lower than a study of Japanese male office workers who were all university graduates $(N=190$; mean $\pm S D, 3.72 \pm 0.68)(20)$. The ability of our findings to generalize should be carefully considered based on these sample characteristics. Second, both measures of HL were based on self-report questionnaires. These responses were the participants' own perceptions of their capabilities and might have been different from objective measures of their abilities. Third, we were unable to examine the influence of HL beyond families that were not cohabiting. Future studies should consider the HL of an individual's entire social network, including family members living apart, friends, and the community.

\section{CONCLUSIONS}

Despite these limitations, this study explored associations among HL within families and showed that HL is distributed within the family. Limited individual ability to achieve health-related tasks might be compensated for by the higher ability of other family members. In addressing problems with limited health 
literacy, future studies should focus not only on the individual but also on people who can provide an individual with support. Further study is needed to find ways to improve HL at the population level, which would complement previously elucidated interventions targeted at the HL of an individual.

\section{DATA AVAILABILITY}

The datasets generated for this study are available on request to the corresponding author.

\section{ETHICS STATEMENT}

This study was approved by the ethical review committee at the University of Tokyo Graduate School of Medicine (approval no. 11476). Respondents who met the inclusion criteria were randomly invited to participate via fax/mail, and those who

\section{REFERENCES}

1. Nutbeam D. Health promotion glossary. Health Promot Int. (1998) 13:349-64. doi: 10.1093/heapro/13.4.349

2. Barry MM, D'Eath M, Sixsmith J. Interventions for improving population health literacy: insights from a rapid review of the evidence. J Health Commun. (2013) 18:1507-22. doi: 10.1080/10810730.2013.840699

3. Dennis S, Williams A, Taggart J, Newall A, Denney-Wilson E, Zwar N, et al. Which providers can bridge the health literacy gap in lifestyle risk factor modification education: a systematic review and narrative synthesis. BMC Fam Pract. (2012) 13:44. doi: 10.1186/1471-2296-13-44

4. Manafo E, Wong S. Health literacy programs for older adults: a systematic literature review. Health Educ Res. (2012) 27:947-60. doi: 10.1093/her/cys067

5. Schaefer CT. Integrated review of health literacy interventions. Orthop Nurs. (2008) 27:302-17. doi: 10.1097/01.NOR.0000337283.55670.75

6. Sheridan SL, Crespo E. Does the routine use of global coronary heart disease risk scores translate into clinical benefits or harms? A systematic review of the literature. BMC Health Serv Res. (2008) 8:60. doi: 10.1186/1472-6963-8-60

7. Taggart J, Williams A, Dennis S, Newall A, Shortus T, Zwar N, et al. A systematic review of interventions in primary care to improve health literacy for chronic disease behavioral risk factors. BMC Fam Pract. (2012) 13:49. doi: 10.1186/1471-2296-13-49

8. Nutbeam D, McGill B, Premkumar P. Improving health literacy in community populations: a review of progress. Health Promot Int. (2017) 33:901-91. doi: 10.1093/heapro/dax015

9. Arozullah AM, Lee SY, Khan T, Kurup S, Ryan J, Bonner M, et al. The roles of low literacy and social support in predicting the preventability of hospital admission. J Gen Intern Med. (2006) 21:140-5. doi: 10.1007/s11606-006-0248-z

10. Lee SY, Arozullah AM, Cho YI. Health literacy, social support, and health: a research agenda. Soc Sci Med. (2004) 58:1309-21. doi: 10.1016/S0277-9536(03)00329-0

11. Papen U. Literacy, learning and health - A social practices view of health literacy. Liter Numer Stud. (2009) 16:19-34. doi: 10.5130/lns.v0i0.1275

12. Edwards M, Wood F, Davies M, Edwards A. 'Distributed health literacy': longitudinal qualitative analysis of the roles of health literacy mediators and social networks of people living with a long-term health condition. Health Expect. (2015) 18:1180-93. doi: 10.1111/hex.12093

13. Dutta MJ, Kaur S, Luk P, Lin J, Lee ST. Health Information Seeking Among Singaporeans: roles and collective contexts. Health Commun. (2017) 33:43342. doi: 10.1080/10410236.2016.1278493

14. Sørensen K, Van den Broucke S, Pelikan JM, Fullam J, Doyle G, Slonska Z, et al. Measuring health literacy in populations: illuminating the design and development process of the European Health Literacy agreed to participate in the survey were asked to provide a completed consent form.

\section{AUTHOR CONTRIBUTIONS}

HI contributed to conceptualization and design of the study, analysis, interpretation of data, and drafted the manuscript. TK contributed to conceptualization and design of the study and interpretation of data. All authors contributed to critical revision of the manuscript for important intellectual content, read, and approved the final manuscript.

\section{FUNDING}

This study was supported by JSPS KAKENHI Grant Number $16 \mathrm{H} 05211$.

Survey Questionnaire (HLS-EU-Q). BMC Publ Health. (2013) 13:948. doi: 10.1186/1471-2458-13-948

15. Sørensen K, Van den Broucke S, Fullam J, Doyle G, Pelikan J, Slonska $\mathrm{Z}$, et al. Health literacy and public health: a systematic review and integration of definitions and models. BMC Publ Health. (2012) 12:80. doi: 10.1186/1471-2458-12-80

16. Duong TV, Aringazina A, Baisunova G, Nurjanah, Pham TV, Pham $\mathrm{KM}$, et al. Measuring health literacy in Asia: Validation of the HLS-EUQ47 survey tool in six Asian countries. J Epidemiol. (2017) 27:80-86. doi: 10.1016/j.je.2016.09.005

17. Nakayama K, Osaka W, Togari T, Ishikawa H, Yonekura Y, Sekido A, et al. Comprehensive health literacy in Japan is lower than in Europe: a validated Japanese-language assessment of health literacy. Bmc Publ Health. (2015) 15:505. doi: 10.1186/s12889-0151835-x

18. Gerich J, Moosbrugger R. Subjective estimation of health literacy-what is measured by the HLS-EU scale and how is it linked to empowerment? Health Commun. (2018) 33:254-63. doi: 10.1080/10410236.2016.1255846

19. Sørensen K, Pelikan JM, Röthlin F, Ganahl K, Slonska Z, Doyle G, et al. et al. Health literacy in Europe: comparative results of the European health literacy survey (HLS-EU). Eur J Publ Health. (2015) 25:1053-8. doi: 10.1093/eurpub/ckv043

20. Ishikawa $H$, Nomura $K$, Sato $M$, Yano E. Developing a measure of communicative and critical health literacy: a pilot study of Japanese office workers. Health Promot Int. (2008) 23:269-74. doi: 10.1093/heapro/dan017

21. Nutbeam D. Health literacy as a public health goal: a challenge for contemporary health education and communication strategies into the $21 \mathrm{st}$ century. Health Promot Int. (2000) 15:259-67. doi: 10.1093/heapro/15.3.259

22. Nutbeam D. The evolving concept of health literacy. Soc Sci Med. (2008) 67:2072-8. doi: 10.1016/j.socscimed.2008.09.050

23. Ishikawa $\mathrm{H}$, Yano $\mathrm{E}$. Patient health literacy and participation in the health-care process. Health Expect. (2008) 11:113-22. doi: 10.1111/j.1369-7625.2008.00497.x

24. Friedman EM, Mare RD. The schooling of offspring and the survival of parents. Demography. (2014) 51:1271-93. doi: 10.1007/s13524-0140303-z

25. Yahirun JJ, Sheehan CM, Hayward MD. Adult children's education and changes to parents' physical health in Mexico. Soc Sci Med. (2017) 181:93-101. doi: 10.1016/j.socscimed.2017. 03.034

26. De Neve JW, Kawachi I. Spillovers between siblings and from offspring to parents are understudied: a review and future directions for research. Soc Sci Med. (2017) 183:56-61. doi: 10.1016/j.socscimed.2017. 04.010 
27. Nielsen-Bohlman L, Panzer AM, Kindig DA. Health Literacy: A Perscription to End Confusion. Washington, DC: The National Academies Press (2004).

28. Paasche-Orlow MK, Wolf MS. The causal pathways linking health literacy to health outcomes. Am J Health Behav. (2007) 31(Suppl 1):S19-26. doi: 10.5993/AJHB.31.s1.4

29. Waverijn G, Heijmans M, Spreeuwenberg P, Groenewegen PP. Associations between neighborhood social capital, health literacy, and self-rated health among people with chronic illness. J Health Commun. (2016) 21(sup2):36-44. doi: 10.1080/10810730.2016.1179369

30. Kim YC, Lim JY, Park K. Effects of health literacy and social capital on health information behavior. J Health Commun. (2015) 20:1084-94. doi: 10.1080/10810730.2015.1018636

31. Kawachi I. Social capital and community effects on population and individual health. Ann NY Acad Sci. (1999) 896:120-30. doi: 10.1111/j.1749-6632.1999.tb08110.x

32. Ratzan SC. Health literacy: communication for the public good. Health Promot Int. (2001) 16:207-14. doi: 10.1093/heapro/16.2.207
33. Nutbeam D, Kickbusch I. Advancing health literacy: a global challenge for the 21st century. Health Promot Int. (2000) 15:183-4. doi: 10.1093/heapro/15.3.183

34. Ishikawa $\mathrm{H}$, Kato M, Kiuchi T. Associations of health literacy and information sources with health-risk anxiety and protective behaviors. I Commun Healthcare. (2016) 9:33-9. doi: 10.1080/17538068.2015.1133004

Conflict of Interest Statement: The authors declare that the research was conducted in the absence of any commercial or financial relationships that could be construed as a potential conflict of interest.

Copyright (c) 2019 Ishikawa and Kiuchi. This is an open-access article distributed under the terms of the Creative Commons Attribution License (CC BY). The use, distribution or reproduction in other forums is permitted, provided the original author(s) and the copyright owner(s) are credited and that the original publication in this journal is cited, in accordance with accepted academic practice. No use, distribution or reproduction is permitted which does not comply with these terms. 